\title{
COMMENTARY
}

\section{Highlighting the paucity of quality data on pediatric stone disease}

\author{
Keara DeCotiis, MD; Martin A. Koyle, MD, MSc \\ Pediatric Urology, The Hospital for Sick Children and University of Toronto, Toronto, ON, Canada
}

Cite as: Can Urol Assoc J 2019;13(10):342. http://dx.doi.org/10.5489/cuaj.6250

See related article on page 334

$\mathrm{P}$ ediatric nephrolithiasis is a rapidly escalating condition worldwide. With larger volumes of younger patients being impacted and as experience with this population is gained, the number of investigative publications on the subject have also begun to escalate.

Cochrane systematic reviews have long been recognized as the highest standard in evidence-based healthcare. The outcome of these reviews is completely dependent on the quality of the input studies evaluated. The Cochrane review performed by Barreto and colleagues demonstrates uncertainty about medical and surgical interventions used to address pediatric stone disease. The republication of this article in the CUAJ highlights for its readers the very lowquality data for both comparison of techniques and outcomes. It also stresses the lack of evidence-based outcomes for guiding patient management.

Traditionally, pediatric stone disease treatment and management has been modeled on urological treatment of the adult patient. Miniaturization of technology has allowed urologists to apply similar techniques of surgical intervention to younger patients. Medications for stent discomfort, urine alkalization, stone passage rates, etc. have likewise been adapted to this group. Thus, it becomes evident that pediatric urologists follow adult guidelines due to the lack of evidence-based pediatric literature. Not unlike other conditions, performing randomized control studies in children can be problematic.

It is important to acknowledge the physiological and anatomical differences between the two populations with

regards to management. The sensitivity of the developing nephron to shockwave lithotripsy, technical challenges of navigating an infant ureter, and issues with visualization and flow during percutaneous nephrolithotomy are just a few of the challenges one faces when deciding how to intervene on a pediatric patient.

The move away from extracorporeal shock wave lithotripsy in this age group follows current trends in North America due to concerns for the developing renal unit. The approach to large stones addressed with percutaneous nephrolithotomy is extremely variable. Treatment may be completed with mini vs. micro technique, tubeless or tubed, with laser or ultrasonic. Risks of stricture or stonefree rates with ureteroscopy are conveyed to parents using data obtained from adults, and pre-stenting is often required due to ureteral diameter. Counselling families using evidence from adult populations can be frustrating for both parties and often leave much to be desired. This is further complicated by the diversity management strategies among pediatric urologists.

This month's Cochrane review highlights the overall paucity of quality data surrounding pediatric stone disease. Ideally, randomized studies with long-term followup are required in order to help establish pertinent guidelines, outcomes data, and suggest the most appropriate techniques when dealing with nephrolithiasis in the pediatric population. As Albert Einstein wrote, "Learning is experience. Everything else is information."

Competing interests: The authors reports no competing personal or financial interests.

Correspondence: Dr. Martin A. Koyle, Pediatric Urology, The Hospital for Sick Children and University of Toronto, Toronto, ON, Canada; martin.koyle@sickkids.ca 